Copyright (C 2014 IEEE. Personal use of this material is permitted. Permission from IEEE must be obtained for all other uses, in any current or future media, including reprinting/republishing this material for advertising or promotional purposes, creating new collective works, for resale or redistribution to servers or lists, or reuse of any copyrighted component of this work in other works. 


\section{A Method to Implement DAISY Online Delivery Protocol}

Azadeh Nazemi

Department of Electrical and Computer Engineering, Curtin University, Perth, WA, Australia Azadeh.nazemi@postgrad.curtin.edu.au
Iain Murray \& David A. McMeekin

Department of Electrical and Computer Engineering, Department of Spatial Sciences,

Curtin University, Perth, WA, Australia

I.murray@curtin.edu.au

D.McMeekin@curtin.edu.au 
Abstract - Digital Accessible Information System format (DAISY) is designed for vision impaired users to access to electronic document. The purpose of this project is creating a reliable and free one-way audio link over the Internet to provide low-cost DAISY format transmission, which support vision impaired users to access and listen DAISY books as audio stream through the link. By connecting to this link, clients can save desired files on their own device as much as they listen and they do not have to download entire DAISY book. This paper describes a method to implement the protocol between user station premises and remote broadcasting library server contains DAISY books. The method explains audio signal streaming from one computer to another, by using free open source software.

Keywords - Digital Accessible Information System (DAISY), Audio Streaming, vision impaired, DAISY Online Delivery Protocol (DODP)

\section{INTRODUCTION}

Currently DAISY readers who are blind, vision impaired or have a print disability can access to reading materials in form of DAISY format in two methods: 1.Online searching for the specific book through the talking book library web service and downloading entire DAISY book contains all text base files and audio files.

2. DAISY book can be made available on demand from the library service for blind.

The Association for the Blind (WA) provides a talking book library services to these people in Australia and throughout the world via its website (http://www.guidedogswa.org). This service is open to any person with either a verifiable print disability, through low vision, physical disability (eg quadriplegia) or learning difficulty (eg Dyslexia). The library contains a collection of 67,640 books as of 27 May 2013 and continues to increase this collection on a daily basis. Current delivery is via several methods: clients physically visit the library and load books onto their daisy player or USB device, books may be posted on a USB cartridge, which is designed for ease of handling by people with limited mobility or dexterity (shown in figure 1)

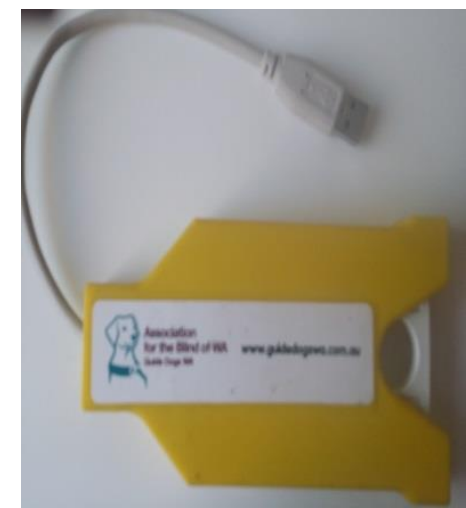

Fig 1. Designed USB cartridge for Blind or limited mobility people

Books may be downloaded from the web site. The download method, whilst useful in many circumstances, has several disadvantages. Firstly, the current methods require the user to download the entire book prior to being able to listen/read it. This is time consuming and tends to consume a significant proportion of download quota (most books are in excess of $300 \mathrm{MB}$ ). Regarding to the Internet access, this is a significant issue. It is worth noting that, many of the library users cannot afford to pay the expense of large quota connections to Internet. In Many cases, they cannot travel to the library on a regular basis due to their conditions. Moreover, this current method does not suit the delivery of temporary material, such as newspapers, catalogues and magazines that have a short period of relevance. DAISY online delivery protocol (DODP) will allow users to download only the sections of DAISY book which they are interested. In the result, significant time and bandwidth usage are saved. For example, a user may wish to read todays newspaper. With the current exist method the full paper must be downloaded. However, DODP proposes the user to specify section such as, the sports section. Then user would be able to immediately start listening and experience a reading session without need for downloading entire material with minimum delay. This method reduces the cost internet usage. The main targets of this project are:

\section{1- Decreasing latency of delivery}

2- Eliminating need to download the full audio book prior to commencement of listening

3- Reducing Dependency to dedicated DAISY Player and platform

\section{PROTOCOL}

A protocol is set of rules that enable two devices to connect and transmit data. Protocols determine how data are transmitted between devices and over networks. They define issues such as error control and data compression methods. A protocol determines the type of error checking to be used, data compression method (if any) and how the sending device will indicate that it has finished a message.

\section{DAISY}

Digital Accessible Information System (DAISY) is a technical standard for digital audio books, periodicals and computerized text. DAISY is designed to be a complete audio substitute for print material and is specifically designed for use by people with print disabilities, including blindness, impaired vision, and dyslexia[1]. Based on MP3 and XML, the format has advanced features in addition to traditional audio book. Users can search, place bookmarks, precisely navigate line by line, and regulate the speaking speed without distortion. DAISY also provides aurally accessible tables, references and additional information. As a result, DAISY allows visually impaired listeners to navigate something as complex as an encyclopaedia or textbook, otherwise impossible using conventional audio recordings. In the DAISY standard, navigation is enabled within a sequential and hierarchical structure consisting of (marked-up) text synchronized with audio. DAISY 2 was based on XHTML and SMIL. DAISY 3 is a newer technology, also based on XML, and is standardized as ANSI/NISO Z39.862005 


\section{STREAMING}

Streaming is used for sending data, usually audio or video, in a way that allows it to start being processed before it is completely received [2]. A user receiver/player can begin playing the data (such as mp3) before the entire file has been transmitted. Streaming uses a streaming protocol to control the data transfer and prevents bandwidth waste because the received packets do not add up to a file. Streaming can be broadly divided into on-demand and real-time categories. With on-demand streaming, the client requests an audio streaming to receive it. With real-time streaming, the sender determines what to send, and the receiver plays it back as it is received, with a slight and consistent delay. With on-demand streaming, the service has to open files as they are requested and keep streams going to each client. If the system load is heavy, it may have to juggle many separate streams and it may fall behind, so that clients are sometimes forced to pause. This is annoying but still considered acceptable, as long as these interruptions are held to an absolute minimum. Streaming servers commonly support more than one protocol allowing them to fall back on alternative protocols if the first choice fails.

\section{DAISY STREAMING ONLINE ON-DEMAND PROTOCOL}

The DAISY Consortium has developed the global standard on online book distribution. Streaming and downloading are made possible on various book readers/players like talking book players, mobile devices, personal computers, etc. Many libraries serving the visually impaired have started an online book distribution service by using the DAISY Online Delivery Protocol (DODP) [3]. The DODP allows computer-free online distribution directly to a talking book player. When users call a library for books of their interest, they will have access to the books using their identification (ID). Some libraries or organizations regularly and automatically distribute Newspapers or magazines that users subscribe [4].DAISY streaming is a protocol for distributing DAISY books. It identifies content by URL and is designed to integrate seamlessly with the web. The DAISY streaming advantage is that the server can support multiple requests of the same file, which are received concurrently. There is a possibility that the server may receive many requests for a single file from different users. It is used for establishing and controlling file transfer between server and clients. In general broadcasting an audio signal from a single source to a larger audience, does not need to be able to communicate back to the source (i.e. the audience listens only).The DAISY streaming protocol would be adaptable to multiple playback devices including iOS devices (iPhone, iPad and iPod), Android tablets and phones along with desktop computers. The DAISY streaming protocol aims to take advantage of mobile phone devices that almost all people have already even older and non-technical users.

\section{IMPLANTATION}

The protocol specifies how a reading system (player) should communicate with a service (content provider). Both the reading system and service must support the DODP.
In order to connect to a service the reading system must have a web address for the service and a username and password which to $\log$ on. The service provider would provide these pieces of information. Communications between the reading system and service use Secure Hyper Text Transfer Protocol (HTTP ).

The protocol allows the service to describe itself to the reading system and for the reading system to describe its capabilities to the service. This allows the service to only provide features that the reading system can present to the user. For example if the reading system has no way for the user to enter text then the service will not ask the user to do so[5].

Based on this protocol, an ordinary broadcast web server, a static map for library bookshelves, and the end user command sender with authority are main entities for a DAISY streaming file distribution system.

A user must be able to play and pause, to facilitate realtime playback control of mp3 audio streaming files related to specific parts of a certain book from the server; Run navigation commands; and send request to server

The important factors for the server are being robust and fast, having access to library book shelves and potential for traffic handling.

The implementation method which has described in this paper will use HTTP protocol for all communication between the client and the server for mp3 audio streaming. HTTP protocol usually uses port 80 or 8080 . The SSH protocol will be used to transfer xml files from the server to the client and for sending requests from the client to the server. The SSH protocol can be used as a generic transport layer encryption mechanism, providing both host authentication and user authentication, together with privacy and integrity protection. $\mathrm{SSH}$ is inherently secure and changing the port used for SSH is a relatively simple procedure. SSH provides secure login, file transfer, X11, and TCP/IP connections over an untrusted network. It uses cryptographic authentication, automatic session encryption, and integrity protection for transferred data [6].

\section{A. Implementation using ICECAST2, ICES}

Nowadays internet audio streaming is a well-established concept. Icecast 2 is an open source server software for streaming multimedia. The setup is intended to be a one-way pipe for high quality live audio signal. Icecast 2 is used on the server as a connection point where audio content can be streamed from to different locations. The audio stream can be shared to hundreds of listeners via the Internet. In order to play the audio the Icecast compatible client software needs to be running on the client such as mpg123 and mplayer which are used as a player. Ices is an application that can be used as a streaming source, running on the server or source client. Icecast 2 is a web server that clients (like iTunes, or a browser) connect to in order to receive the streaming audio signal. Ices and Icecast 2 do not have to run on the same server so that the mp3's come from one machine and then get streamed up to Icecas 2 server, which in turn streams out to multiple listeners on the internet. For the following implementation example, 
both Ices and Icecast 2 will be running on the same Linux machine.

\section{B. Preparation for Serving Session}

To start serving, a host needs to execute the following steps:

1) Install an icecst 2 server and an ices mp3 source provider on the server machine

2) Start running an icecast 2 server

3) Provide an access list for each registered user considering the area fields in their profile

4) Control user authentication by checking user profile

5) Start running the ices service to load user requested mp3 files to the server port

6) Investigate user requests for access to individual books and send the user back the book maps xml files *.ncx ${ }^{*}$.opf for ANSI/NISO Z39.86-2005 DAISY or ncc .html for Daisy 2.02 (all of these file transmissions use SSH protocol over the internet)

7) Put requested mp3 files on the server idle port using HTTP protocol

\section{Preparation Reading Session}

To start reading session the following: steps must be performed:

1- Install mplayer or another audio streaming player on device

2- Register with a local or international online DAISY library and create profile

3- Install DAISY streaming application on device

4- Connect to library server by running the DAISY streaming application and enter their user name and password

5- Run DAISY streaming application

6- Connect to library server

7- Login to server by entering username and password

8- Access list browsing and select desired book

9- Receive the book maps xml files *.ncx ,*.opf for ANSI/NISO Z39.86-2005 DAISY or ncc .html for Daisy 2.02 from the server through SSH protocol

10-Navigate through different parts of a book and select a part using installed DAISY Player application in own device

11-Send a request for a mp3 file related to the selected part of chosen book to server by modifying own playlist.txt and Ices configuration and send them to server through $\mathrm{SSH}$

12-Play back mp3 audio streaming using HTTP protocol

13-Record audio streaming simultaneously with playback instead of downloading to prevent increased usage of download quota on user device, provide opportunity for the server to be able support many users and respond to many unique requests simultaneously

\section{Library Bookshelves}

Each online library consists of various book categories which are saved in the storage. The must have access to this storage directly. All books are saved in the storage based on categories. Users access to books according to their profile information. When the user modifies the access area information in their profile the server provides new key access

\section{E. Implementation Requirements and configuration}

The basic concept for streaming mp3's has three steps , contains putting mp3's on cloud server, setting up a streaming audio server and using a web client that points to the cloud server. Therefore the requirements are:

1- LINUX based computer as an audio link server computer

2- Icecast 2 as a streaming software backend for the server computer

3- mpg123 an audio stream player for the listener client

4- Ices (Linux )as mp3 audio streaming application for the source client computer which can be installed on server as well.

To configure LAN client, comment out the line iface eth0 inet dhcp by adding\#

\begin{tabular}{l} 
sudo nano /etc/network/interfaces \\
auto eth0 \\
\#iface eth0 inet dhcp \\
ifup eth0 \\
\hline
\end{tabular}

To configure LAN server add manual IP configuration with following new lines:

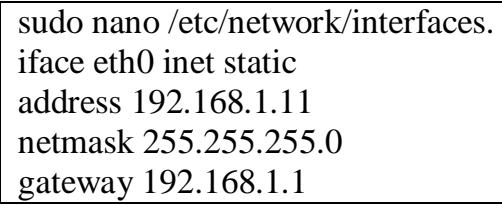

To install Ices:Download the ices0 source package from icecast.org

./configure
Make
sudo make install

Set up an Ices configuration file to tell Ices where to find mp3's

\begin{tabular}{|l|}
\hline mkdir /etc/ices/cd /etc/ices \\
$\mathrm{cp} /$ usr/local/etc/ ices.conf.dist \\
sudo apt-get install icecast2 \\
cat/etc/default/icecast2 \\
sudo nano/etc/default/icecast2 \\
\hline
\end{tabular}

Change the line ENABLE=false to ENABLE=true. 
Put a playlist file called "playlist.txt" in the same directory as the ices.conf. The playlist.txt file is just a plain text file with the full path of each mp3.

To setup and configure icecast2 server(By default Icecast2 is disabled)

\begin{tabular}{l}
\hline sudo apt-get install icecast2 \\
cat /etc/default/icecast2 \\
sudo nano /etc/default/icecast2 \\
Change the line ENABLE=false ENABLE=true. \\
\hline sudo nano /etc/icecast2/icecast.xml \\
authentication:source-password = [chosen \\
password],relay-password = [chosen password], \\
admin-user = [admin username],admin-password \\
$=[$ chosen password]
\end{tabular}

To start icecast 2

sudo /etc/init.d/icecast2 starts
sudo icecast2 -c

To stop the server icecast2

sudo /etc/init.d/icecast2 stop

To make the Icecast 2 server run in the background. sudo icecast2 -b -c /etc/icecast2/icecast.xml

In a web browser from another computer in the same network or in the server to shows server status http://server-ip- address:8000

To listen or play MP3 stream on client deviece mplayer http://localhost: 8000

To capture an MP3 s and save a stream using wget: wget http://host:8000 -O download01.mp3.

But capture audio streaming by this method make double download quota usage. The proposed method to save what user wants and what user listens is recording stream data from sound card as long as it plays. This command shows the defaults for Icecast, including path to server configuration file, and user ID and group that are used for running the Icecast 2 server..

\section{VII.DAISY STREAMING PROCEDURE BASED ON ICECAST2 SERVER \& ICES SOURCE CLIENT}

The figure 2 illustrates DAISY streaming procedure.

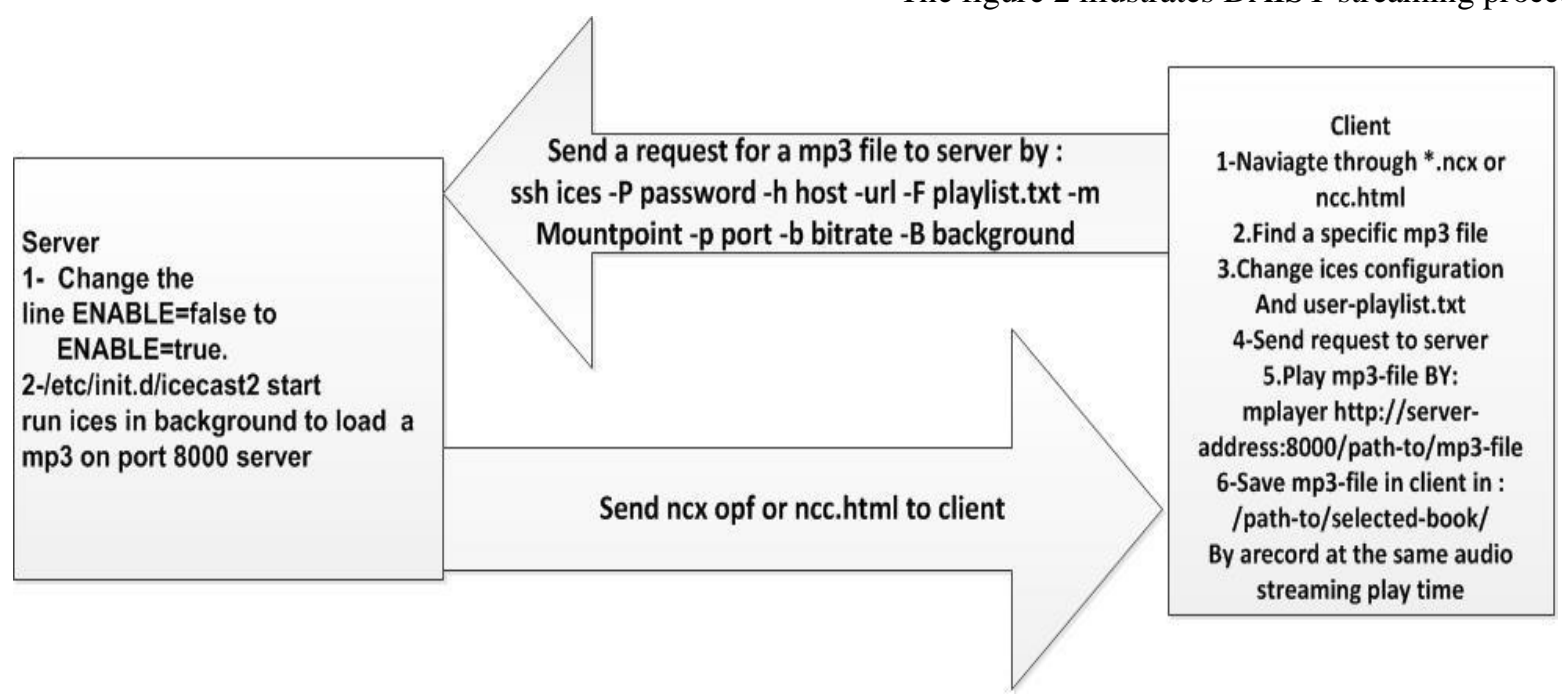

Fig. 2. DAISY streaming procedure

The user device connects to library server using internet connection. After passing user authentication step through username and password user will be able to search through the online catalogue for specific material and select one. Installed DAISY streaming application on user device automatically make a folder in storage device /pathto/selected-book to save what listen and send a request to server for XML file. Server sends ncc.html or .ncx and .opf files to user device and saves it in as a book map and makes a temporary folder /path-to/user-folder to keep the user information such as playlist
DAISY streaming application on user device having map file will be able to navigate through different levels of book such as chapters, section and etc..

When the desired part of book has been found, the user device makes a request for $\mathrm{mp} 3$ file related to this part by appending path-to-mp3/mp3-file-name.mp3 to the playlist.txt and edits ices.conf by changing the ICES configuration . Send a request for a mp3 file to server by: 
ssh ices -P password d -h host -url -F user- playlist.txt -m Mountpoint -p port -b bitrate -B background

scp user-playlist.txt root@ server-address:/path-to/user -

folder/user-playlist .txt

ICECAST2 on Server automatically put the requested mp3 file on considered port as soon as changing the ICES configuration and playlist.txt are occurred and by result streaming will be started. As soon as audio streaming starts a timer starts to keep the elapsed time audio codec in user device and DAISY streaming application starts to record and save it, so as much as user listening to mp3 file it can be recorded and saved, so streaming and recording are sync. Sending from server to user device using capture command such as "wget" command causes to download unwanted data , increase download data usage and waste band width.

During audio streaming if the user presses pause, the user device kills the play audio stream as well as the recording stream on the device and saves elapsed time. Then when the resume button is pressed audio streaming and recording start from this point instead of mp3 commencing from the beginning point again.

During online connection if the user presses exit and wants to finish the reading session, all information including the complete path and mp3 file name, time elapsed are appended to bookmark.dat and at beginning of next session for the same book, the bookmark table is sent to the server and kept in /path-to/user -folder

\section{VIII.CONCLUSION}

The research undertaken performs DODP implementation in small scale successfully with optimal speed and minimum download internet usage. Further investigation needs to design and develop DAISY streaming application for user device to solve remain issues regarding bookmarking and searching ability inside local bookmark table.

\section{REFERENCES}

[1] Sabine Tenta.2009. The Audible Gate to the World: The West German Audio Book Library for the Blind (Goethe-Institut, 2009)

[2] Gary McGath, Basics of streaming protocols.2013

Available at: http://www.garymcgath.com/streamingprotocols.html, Last updated 29-May-2013.

[3] DAISY Online Delivery Project, Available at: http://www.daisy.org/project/daisy-online-delivery last retrieved :18 December 2013

[4] Specification for the DAISY Online Delivery Protocol: , Available at : http://www.daisy.org/projects/daisy-onlinedelivery/drafts/20100402/do-spec-20100402.html last retrieved :15 January 2014

[5] Nick Williamson, DAISY Online Delivery. Available at: http://www.daisy.org/daisypedia/daisy-online-delivery last retrieved :1 February 2014

[6] Barrett, Daniel; Richard E. Silverman (2001), SSH, The Secure Shell: The Definitive Guide, Cambridge: O'Reilly, ISBN 0-596-00011- 
